\title{
HADAMARD PRODUCTS OF CERTAIN MEROMORPHIC UNIVALENT FUNCTIONS
}

\author{
OH SANG KWON* AND NAK EUN CHO**
}

\begin{abstract}
The object of the present paper is to show convolution properties, order of starlikeness, integral transforms and the extreme points for certain classes of meromorphic univalent functions having postive coefficients. All of the results are sharp.
\end{abstract}

\section{Introduction}

Let $\Sigma$ denote the class of functions of the form

$$
f(z)=\frac{1}{z}+\sum_{k=1}^{\infty} a_{k} z^{k}
$$

which are regular and univalent in the punctured disk $E=\{z: 0<|z|<1\}$. A function $f$ belonging to $\Sigma$ is said to be meromorphically starlike of order $\alpha$ if it satisfies

$$
-\operatorname{Re}\left\{\frac{z f(z)}{f(z)}\right\}>\alpha
$$

for some $\alpha(0 \leq \alpha<1)$ and all $z \in U=\{z:|z|<1\}$. We denote by $\Sigma^{*}(\alpha)$ the class of all meromorphically starlike functions of order $\alpha$.

The class $\Sigma^{*}(\alpha)$ and related classes have been extensively studied by Bajpai[2], Clunie [4], Pommerenke [7], Morgra, Reddy and Juneja [6] and others ([1], [3]).

The Hadamard product or convolution of two functions $f, g$ in $\Sigma$ will be denoted by $f * g$. Robertson [8] has shown that if $f, g \in \Sigma$, then so is their convolution $f * g$.

Let $\Sigma(\lambda, \alpha, \beta, \gamma)$ denote the class of functions $f$ in $\Sigma$ satisfying the condition

$$
\operatorname{Re}\left|z^{2}\left(D^{\lambda} f(z)\right)^{\prime}+1\right|<\beta\left|(2 \gamma-1) z^{2}\left(D^{\lambda} f(z)\right)^{\prime}+(2 \alpha \gamma-1)\right|
$$

for some $\alpha(0 \leq \alpha<1), \beta(0<\beta \leq 1), \gamma\left(\frac{1}{2} \leq \gamma \leq 1\right)$ and for all $z \in U$, where $D^{\lambda}: \Sigma \rightarrow \Sigma$ is the operator defined by

$$
D^{\lambda} f(z)=\frac{1}{z(1-z)^{\lambda+1}} * f(z)(\lambda>-1)
$$

Received December 24, 1996.

1991 Mathematics Subject Classification. 30C45.

Key words and phrases. Convolution properties, order of starlikeness, integral transforms, extreme points. 
Notice that from the identity

$$
\frac{1}{z(1-z)^{\lambda+2}}=\frac{1}{z(1-z)^{\lambda+1}} *\left(\frac{\lambda+2}{(\lambda+1) z(1-z)}+\frac{2 z-1}{(\lambda+1) z(1-z)^{2}}\right)
$$

we get

$$
z\left(D^{\lambda} f(z)\right)^{\prime}=(\lambda+1) D^{\lambda+1} f(z)-(\lambda+2) D^{\lambda} f(z)(\lambda>-1) .
$$

For $\lambda=n \in N_{0}=\{0,1,2, \ldots\}$, we note [9] that the relation (1.2) may be expressed as

$$
D^{n} f(z)=\frac{1}{z}\left(\frac{z^{n+1} f(z)}{n !}\right)^{(n)} .
$$

Let $\Sigma_{p}, \Sigma_{p}^{*}(\alpha)$ and $\Sigma_{p}(\lambda, \alpha, \beta, \gamma)$ denote the subclasses of $\Sigma, \Sigma^{*}(\alpha)$ and $\Sigma(\lambda, \alpha, \beta, \gamma)$, respectively, whose elements can be expressed in the form

$$
f(z)=\frac{1}{z}+\sum_{k=1}^{\infty} a_{k} z^{k} \quad\left(a_{k} \geq 0, z \in U\right) .
$$

In particular, the classes $\Sigma_{p}(0, \alpha, 1,1)$ and $\Sigma_{p}(0, \alpha, \beta, \gamma)$ are introduced by Aouf[1] and Cho, Lee and $\mathrm{Owa}[3]$, respectively.

For the classes $\Sigma_{p}(0, \alpha, \beta, \gamma)$ and $\Sigma_{p}^{*}(\alpha)$, Cho, Lee and Owa[3] and Mogra, Reddy and Juneja [6] proved the following results.

Theorem $\mathbb{A}$. Let $f$ be of the form (1.5) and $0 \leq \alpha<1,0<\beta \leq 1, \frac{1}{2} \leq \gamma \leq 1$. Then $f \in \Sigma_{p}(0, \alpha, \beta, \gamma)$ if and only if

$$
\sum_{k=1}^{\infty} k(1+2 \beta \gamma-\beta) a_{k} \leq 2 \beta \gamma(1-\alpha) .
$$

Theorem $\mathbb{B}$. The extreme points of $\Sigma_{p}(0, \alpha, \beta, \gamma)\left(0 \leq \alpha<1,0<\beta \leq 1, \frac{1}{2} \leq \gamma \leq 1\right)$ are the functions given by $f_{0}(z)=\frac{1}{z}$ and

$$
f_{k}(z)=\frac{1}{z}+\frac{2 \beta \gamma(1-\alpha)}{k(1+2 \beta \gamma-\beta)} z^{k} \quad(k=1,2, \ldots) .
$$

Theorem $\mathbb{C}$. Let $f$ be of the form (1.5) and $0 \leq \alpha<1$. Then $f \in \Sigma_{p}^{*}(\alpha)$ if and only if

$$
\sum_{k=1}^{\infty}(k+\alpha) a_{k} \leq 1-\alpha
$$

In this paper, we obtain certain properties of $f * g$ when $f \in \Sigma_{p}(\lambda, \alpha, \beta, \gamma)$ and $g \in \Sigma_{p}(\mu, \delta, \beta, \gamma)$ for $\lambda, \mu \geq 0$ and $0 \leq \alpha<1,0 \leq \delta<1,0<\beta \leq 1, \frac{1}{2} \leq \gamma \leq 1$. We also 
determine the order of starlikeness and consider integral transforms for the functions in $\Sigma_{p}(\lambda, \alpha, \beta, \gamma)$. Further, we find extreme points of the class $\Sigma_{p}(\lambda, \alpha, \beta, \gamma)$.

\section{Convolution Properties}

We first prove the following result which will be used heavily in the paper.

Lemma 1 . Let $f$ of the form (1.5) be regular in $U, \lambda>-1,0 \leq \alpha<1,0<\beta \leq 1$ and $\frac{1}{2} \leq \gamma \leq 1$. Then $f \in \Sigma_{p}(\lambda, \alpha, \beta, \gamma)$ if and only if

$$
\sum_{k=1}^{\infty} k(1+2 \beta \gamma-\beta) B_{k}(\lambda) a_{k} \leq 2 \beta \gamma(1-\alpha)
$$

where

$$
B_{k}(\lambda)=\frac{(\lambda+1)(\lambda+2) \cdots(\lambda+k+1)}{(k+1) !}
$$

Proof. Since

$$
D^{\lambda} f(z)=\frac{1}{z}+\sum_{k=1}^{\infty} B_{k}(\lambda) a_{k} z^{k}
$$

in view of (1.3) and the definition of $\Sigma_{p}(\lambda, \alpha, \beta, \gamma)$, we have

$$
f \in \Sigma_{p}(\lambda, \alpha, \beta, \gamma) \Longleftrightarrow D^{\lambda} f \in \Sigma_{p}(0, \alpha, \beta, \gamma),
$$

and so Lemma 1 follows immediately from Theorem $\mathrm{A}$.

Corollary 1. Let $f$ be of the form (1.5), $\lambda>-1,0 \leq \alpha<1,0<\beta \leq 1$ and $\frac{1}{2} \leq \gamma \leq 1$. If $f \in \Sigma_{p}(\lambda, \alpha, \beta, \gamma)$, then

$$
a_{k} \leq \frac{2 \beta \gamma(1-\alpha)}{k(1+2 \beta \gamma-\beta) B_{k}(\lambda)}(k \geq 1),
$$

with equality for the functions of the form

$$
F_{k}(z)=\frac{1}{z}+\frac{2 \beta \gamma(1-\alpha)}{k(1+2 \beta \gamma-\beta) B_{k}(\lambda)} z^{k} .
$$

For the next theorem and its corollaries, we assume that $f$ is given by (1.5) and $g$ by

$$
g(z)=\frac{1}{z}+\sum_{k=1}^{\infty} b_{k} z^{k}\left(b_{k} \geq 0, z \in U\right)
$$

Theorem 1. Let $\lambda \geq 0, \mu \geq 0, \nu=0$ or 1 and $0 \leq \alpha<1,0 \leq \delta<1$. If $f \in \Sigma_{p}(\lambda, \alpha, \beta, \gamma)$ and $g \in \Sigma_{p}(\mu, \delta, \beta, \gamma)$, then $f * g \in \Sigma_{p}(\nu, \rho, \beta, \gamma)$, where

$$
\rho=\frac{(1+2 \beta \gamma-\beta)(\lambda+1)(\lambda+2)(\mu+1)(\mu+2)-4 \beta \gamma(1-\alpha)(1-\delta)(\nu+1)(\nu+2)}{(1+2 \beta \gamma-\beta)(\lambda+1)(\lambda+2)(\mu+1)(\mu+2)}
$$


Proof. We need to find the largest $\rho=\rho(\alpha, \delta, \lambda, \mu, \nu, \beta, \gamma)$ for which

$$
\sum_{k=1}^{\infty} B_{k}(\lambda) \frac{k(1+2 \beta \gamma-\beta)}{2 \beta \gamma(1-\alpha)} a_{k} \leq 1
$$

and

$$
\sum_{k=1}^{\infty} B_{k}(\mu) \frac{k(1+2 \beta \gamma-\beta)}{2 \beta \gamma(1-\delta)} b_{k} \leq 1
$$

imply that

$$
\sum_{k=1}^{\infty} B_{k}(\nu) \frac{k(1+2 \beta \gamma-\beta)}{2 \beta \gamma(1-\rho)} a_{k} b_{k} \leq 1
$$

Using the Cauchy-Schwarz inequality, (2.7) and (2.8) together yield

$$
\sum_{k=1}^{\infty} \frac{k(1+2 \beta \gamma-\beta)}{2 \beta \gamma}\left(\frac{B_{k}(\lambda) B_{k}(\mu)}{(1-\alpha)(1-\delta)}\right)^{\frac{1}{2}} \sqrt{a_{k} b_{k}} \leq 1 .
$$

Thus it is sufficient to show that

$$
\frac{B_{k}(\nu)}{1-\rho} a_{k} b_{k} \leq\left(\frac{B_{k}(\lambda) B_{k}(\mu)}{(1-\alpha)(1-\delta)}\right)^{\frac{1}{2}} \sqrt{a_{k} b_{k}}
$$

for all $\rho \leq \rho(\alpha, \delta, \lambda, \mu, \nu, \beta, \gamma)$ and each $k \geq 1$. Equivalently, we need to prove that

$$
\sqrt{a_{k} b_{k}} \leq\left(\frac{B_{k}(\lambda) B_{k}(\mu)}{(1-\alpha)(1-\delta)}\right)^{\frac{1}{2}} \frac{1-\rho}{B_{k}(\nu)}
$$

for all $\rho \leq \rho(\alpha, \delta, \lambda, \mu, \nu, \beta, \gamma)$ and each $k \geq 1$. On the other hand, it follows from (2.10) that

$$
\sqrt{a_{k} b_{k}} \leq \frac{2 \beta \gamma}{k(1+2 \beta \gamma-\beta)}\left(\frac{(1-\alpha)(1-\delta)}{B_{k}(\lambda) B_{k}(\mu)}\right)^{\frac{1}{2}}
$$

for each $k \geq 1$. Therefore, in view of (2.11) and (2.12), it is sufficient to establish that

$$
\frac{2 \beta \gamma}{k(1+2 \beta \gamma-\beta)}\left(\frac{(1-\alpha)(1-\delta)}{B_{k}(\lambda) B_{k}(\mu)}\right)^{\frac{1}{2}} \leq\left(\frac{B_{k}(\lambda) B_{k}(\mu)}{(1-\alpha)(1-\delta)}\right)^{\frac{1}{2}} \frac{1-\rho}{B_{k}(\nu)}
$$

that is, that

$$
\frac{2 \beta \gamma(1-\alpha)(1-\delta) B_{k}(\nu)}{k(1+2 \beta \gamma-\beta) B_{k}(\lambda) B_{k}(\mu)} \leq 1-\rho
$$

which is equivalent to

$$
\rho \leq 1-\frac{2 \beta \gamma(1-\alpha)(1-\delta) B_{k}(\nu)}{k(1+2 \beta \gamma-\beta) B_{k}(\lambda) B_{k}(\mu)}
$$


Note that

$$
B_{k}(\nu)= \begin{cases}1 & \text { if } \nu=0 \\ k+2 & \text { if } \nu=1\end{cases}
$$

Also

$$
\frac{1}{B_{k}(\lambda)} \leq 1, \frac{1}{B_{k}(\mu)} \leq 1
$$

Thus it follows that the right hand side of (2.13) is an increasing function of $k \geq 1$. Setting $k=1$ in (2.13), we have

$$
\begin{aligned}
\rho & \leq 1-\frac{2 \beta \gamma(1-\alpha)(1-\delta) B_{1}(\nu)}{(1+2 \beta \gamma) B_{1}(\lambda) B_{1}(\mu)} \\
& =\frac{(1+2 \beta \gamma-\beta)(\lambda+1)(\lambda+2)(\mu+1)(\mu+2)-4 \beta \gamma(1-\alpha)(1-\delta)(\nu+1)(\nu+2)}{(1+2 \beta \gamma-\beta)(\lambda+1)(\lambda+2)(\mu+1)(\mu+2)}
\end{aligned}
$$

This completes the proof of Theorem 1. The result is sharp for the functions

$$
f(z)=\frac{1}{z}+\frac{4 \beta \gamma(1-\alpha)}{(1+2 \beta \gamma-\beta)(\lambda+1)(\lambda+2)} z \in \Sigma_{p}(\lambda, \alpha, \beta, \gamma)
$$

and

$$
g(z)=\frac{1}{z}+\frac{4 \beta \gamma(1-\delta)}{(1+2 \beta \gamma-\beta)(\mu+1)(\mu+2)} z \in \Sigma_{p}(\mu, \delta, \beta \gamma)
$$

Taking $\lambda=\mu=\nu=0$ and $\alpha=\delta$ in Theorem 1 , we have

Corollary 2. If $f, g \in \Sigma_{p}(0, \alpha, \beta, \gamma)$, then $f * g \in \Sigma_{p}\left(0,1-\frac{2 \beta \gamma(1-\alpha)^{2}}{1+2 \beta \gamma-\beta}, \beta, \gamma\right)$.

Putting $\lambda=\mu=\nu=0$ in Theorem 1, we obtain

Corollary 3. If $f \in \Sigma_{p}(0, \alpha, \beta, \gamma)$ and $g \in \Sigma_{p}(0, \delta, \beta, \gamma)(0 \leq \alpha, \delta<1)$, then $f * g \in$ $\Sigma_{p}\left(0,1-\frac{2 \beta \gamma(1-\alpha)(1-\delta)}{1+2 \beta \gamma-\beta}, \beta, \gamma\right)$.

For the next theorem (Theorem 2) and its corollary, we assume that $f$ is given by (1.5), $g$ by (2.5) and $h$ by

$$
h(z)=\frac{1}{z}+\sum_{k=1}^{\infty}\left(a_{k}^{2}+b_{k}^{2}\right) z^{k} \quad(z \in E) .
$$

Theorem 2. Let $\lambda \geq 0,0 \leq \alpha<1,0<\beta \leq 1$ and $\frac{1}{2} \leq \gamma \leq 1$. Then

$$
f, g \in \Sigma_{p}(\lambda, \alpha, \beta, \gamma) \Longrightarrow \frac{1}{2} h \in \Sigma_{p}(\lambda, \eta, \beta, \gamma),
$$

where

$$
\eta=1-\frac{4 \beta \gamma(1-\alpha)^{2}}{(\lambda+1)(\lambda+2)(1+2 \beta \gamma-\beta)} .
$$


The result is sharp.

Proof. Since $f \in \Sigma_{p}(\lambda, \alpha, \beta, \gamma)$, Lemma 1 yields

$$
\sum_{k=1}^{\infty}\left(B_{k}(\lambda) \frac{k(1+2 \beta \gamma-\beta)}{2 \beta \gamma(1-\alpha)}\right)^{2} a_{k}^{2} \leq\left(\sum_{k=1}^{\infty} B_{k}(\lambda) \frac{k(1+2 \beta \gamma-\beta)}{2 \beta \gamma(1-\alpha)} a_{k}\right)^{2} \leq 1
$$

Similarly,

$$
\sum_{k=1}^{\infty}\left(B_{k}(\lambda) \frac{k(1+2 \beta \gamma-\beta)}{2 \beta \gamma(1-\alpha)}\right)^{2} b_{k}^{2} \leq 1
$$

Hence

$$
\sum_{k=1}^{\infty} \frac{1}{2}\left(B_{k}(\lambda) \frac{k(1+2 \beta \gamma-\beta)}{2 \beta \gamma(1-\alpha)}\right)^{2}\left(a_{k}^{2}+b_{k}^{2}\right) \leq 1
$$

We now want to find the largest $\eta=\eta(\alpha, \beta, \gamma, \lambda)$ such that

$$
\sum_{k=1}^{\infty} \frac{1}{2}\left(B_{k}(\lambda) \frac{k(1+2 \beta \gamma-\beta)}{2 \beta \gamma(1-\eta)}\right)\left(a_{k}^{2}+b_{k}^{2}\right) \leq 1
$$

Thus (2.15) implies (2.16) if

$$
\frac{B_{k}(\lambda) k(1+2 \beta \gamma-\beta)}{2 \beta \gamma(1-\eta)} \leq\left(\frac{B_{k}(\lambda) k(1+2 \beta \gamma-\beta)}{2 \beta \gamma(1-\alpha)}\right)^{2}
$$

or equivalently,

$$
\eta \leq 1-\frac{2 \beta \gamma(1-\alpha)^{2}}{B_{k}(\lambda)^{k}(1+2 \beta \gamma-\beta)} .
$$

Since the right-hand side of (2.17) is an increasing function of $k(k \geq 1)$, we have

$$
\eta \leq 1-\frac{4 \beta \gamma(1-\alpha)^{2}}{(\lambda+1)(\lambda+2)(1+2 \beta \gamma-\beta)} .
$$

The result is sharp for the functions

$$
f(z)=g(z)=\frac{1}{z}+\frac{4 \beta \gamma(1-\alpha)}{(1+2 \beta \gamma-\beta)(\lambda+1)(\lambda+2)} z .
$$

For a function $f$ of the form (1.1), it is easy to see that the condition (2.1) provides only a sufficient condition for the function to be the class $\Sigma(\lambda, \alpha, \beta, \gamma)$. More precisely, the function $f \in \Sigma(\lambda, \alpha, \beta, \gamma)$ if

$$
\sum_{k=1}^{\infty} k B_{k}(\lambda)(1+2 \beta \gamma-\beta)\left|a_{k}\right| \leq 2 \beta \gamma(1-\alpha) .
$$

As a consequence of (2.19), we have 
Theorem 3. For $\lambda>-1,0 \leq \alpha<1,0<\beta \leq 1, \frac{1}{2} \leq \gamma \leq 1$ and $a_{k} \geq 0(k \geq 1)$. let

$$
f(z)=\frac{1}{z}+\sum_{k=1}^{\infty} a_{k} z^{k} \in \Sigma_{p}(\lambda, \alpha, \beta, \gamma), g(z)=\frac{1}{z}+\sum_{k=1}^{\infty} b_{k} z^{k}
$$

for $z \in D$. Then

$$
f * g \in \Sigma(\lambda, \alpha, \beta, \gamma) \text { if }\left|b_{k}\right| \leq 1 \quad(k \geq 1)
$$

and

$$
f * g \in \Sigma_{p}(\lambda, \alpha, \beta, \gamma) \text { if } 0 \leq b_{k} \leq 1 \quad(k \geq 1)
$$

Proof. We observe that

$$
\begin{aligned}
\sum_{k=1}^{\infty} k B_{k}(\lambda)(1+2 \beta \gamma-\beta)\left|a_{k} b_{k}\right| & =\sum_{k=1}^{\infty} k B_{k}(\lambda)(1+2 \beta \gamma-\beta) a_{k}\left|b_{k}\right| \\
& \leq \sum_{k=1}^{\infty} k B_{k}(\lambda)(1+2 \beta \gamma-\beta) a_{k} \leq 2 \beta \gamma(1-\alpha)
\end{aligned}
$$

and so, in view of (2.19), it follows that $f * g \in \Sigma(\lambda, \alpha, \beta, \gamma)$. If $0 \leq b_{k} \leq 1$, then the above observation yields the inequality (2.19) which, using Lemma 1, proves that $f * g \in \Sigma_{p}(\lambda, \alpha, \beta, \gamma)$. This completes the proof of Theorem 3.

Corollary 4. Let $f$ and $g$ be as defined in Theorem 3. If $f \in \Sigma_{p}(\lambda, \alpha, \beta, \gamma)$, then

(i) $f * g \in \Sigma(0, \alpha, \beta, \gamma)$ if $\left|b_{k}\right| \leq 1 \quad(k \geq 1)$.

(ii) $f * g \in \Sigma_{p}(0, \alpha, \beta, \gamma)$ if $0 \leq b_{k} \leq 1 \quad(k \geq 1)$.

\section{Order of Starlikeness} then

Theorem 4. Let $\lambda \geq 0,0 \leq \alpha<1,0<\beta \leq 1$ and $\frac{1}{2} \leq \gamma \leq 1$. If $f \in \Sigma_{p}(\lambda, \alpha, \beta, \gamma)$,

$$
f \in \Sigma_{p}^{*}\left(\frac{(1+2 \beta \gamma-\beta)(\lambda+1)(\lambda+2)-4 \beta \gamma(1-\alpha)}{(1+2 \beta \gamma-\beta)(\lambda+1)(\lambda+2)+4 \beta \gamma(1-\alpha)}\right) .
$$

The result is sharp.

Proof. In view of Lemma 1 and Theorem C, it suffices to show that, for a function $f$ of the form (1.5), the inequality (2.1) implies that

$$
\sum_{k=1}^{\infty}\left(\frac{k+\frac{(1+2 \beta \gamma-\beta)(\lambda+1)(\lambda+2)-4 \beta \gamma(1-\alpha)}{(1+2 \beta \gamma-\beta)(\lambda+1)(\lambda+2)+4 \beta \gamma(1-\alpha)}}{1-\frac{(1+2 \beta \gamma-\beta)(\lambda+1)(\lambda+2)-4 \beta \gamma(1-\alpha)}{(1+2 \beta \gamma-\beta)(\lambda+1)(\lambda+2)+4 \beta \gamma(1+\alpha)}}\right) a_{k} \leq 1
$$


which holds if

$$
\frac{k+\frac{(1+2 \beta \gamma-\beta)(\lambda+1)(\lambda+2)-4 \beta \gamma(1-\alpha)}{(1+2 \beta \gamma-\beta)(\lambda+1)(\lambda+2)+4 \beta \gamma(1-\alpha)}}{1-\frac{(1+2 \beta \gamma-\beta)(\lambda+1)(\lambda+2)-4 \beta \gamma(1-\alpha)}{(1+2 \beta \gamma-\beta)(\lambda+1)(\lambda+2)+4 \beta \gamma(1+\alpha)}} \leq B_{k}(\lambda) \frac{k(1+2 \beta \gamma-\beta)}{2 \beta \gamma(1-\alpha)}
$$

or equivalently, when

$$
G(k, \lambda, \alpha, \beta, \gamma)=\frac{(k+1)(1+2 \beta \gamma-\beta)(\lambda+1)(\lambda+2)+4(k-1) \beta \gamma(1-\alpha)}{4 k B_{k}(\lambda)(1+2 \beta \gamma-\beta)} \leq 1 .
$$

We note that $G(1, \lambda, \alpha, \beta, \gamma)=1$. Therefore, it suffices to show that $G(k, \lambda, \alpha, \beta, \gamma)$ is a decreasing function of $k(k \geq 1)$, that is, that

$$
\begin{aligned}
\frac{G(k+1, \lambda, \alpha, \beta, \gamma)}{G(k, \lambda, \alpha, \beta, \gamma)}= & \frac{[(k+2)(1+2 \beta \gamma-\beta)(\lambda+1)(\lambda+2)+4 k \beta \gamma(1-\alpha)]}{[(k+1)(1+2 \beta \gamma-\beta)(\lambda+1)(\lambda+2)+4(k-1) \beta \gamma(1-\alpha)]} \\
& \times \frac{k(k+2)}{(k+1)(\lambda+k+2)} \leq 1 .
\end{aligned}
$$

But the inequality (3.2) holds if and only if, for each fixed $\lambda(\lambda \geq 0)$ and $\alpha(0 \leq \alpha<1)$, $\beta(0<\beta \leq 1)$ and $\gamma\left(\frac{1}{2} \leq \gamma \leq 1\right)$, we have

$$
\begin{aligned}
H(k)= & {[(k+1)(1+2 \beta \gamma-\beta)(\lambda+1)(\lambda+2)+4(k-1) \beta \gamma(1-\alpha)](k+1)(\lambda+k+2) } \\
& -[(k+2)(1+2 \beta \gamma-\beta)(\lambda+1)(\lambda+2)+4 k \beta \gamma(1-\alpha)] k(k+2) \geq 0 .
\end{aligned}
$$

We observe that $H(1)=(4 \lambda+3)(\lambda+1)(\lambda+2)(1+2 \beta \gamma-\beta)-12 \beta \gamma(1-\alpha) \geq 0$ and

$$
\begin{aligned}
H(k+1)-H(k)= & {[2(1+2 \beta \gamma-\beta)(\lambda+1)(\lambda+2)+8 \beta \gamma(1-\alpha)] \lambda k } \\
& +(3 \lambda+1)\left(\lambda^{2}+3 \lambda\right)(1+2 \beta \gamma-\beta)+4 \beta \gamma((3 \lambda+\alpha)+(1-\alpha) \lambda) \\
& +2(1-\beta)(3 \lambda+1) \geq 0 .
\end{aligned}
$$

for all $\lambda \geq 0,0 \leq \alpha<1,0<\beta \leq 1$ and $\frac{1}{2} \leq \gamma \leq 1$. This completes the proof of Theorem 4. The result is sharp for the functions

$$
f(z)=g(z)=\frac{1}{z}+\frac{4 \beta \gamma(1-\alpha)}{(1+2 \beta \gamma-\beta)(\lambda+1)(\lambda+2)} z .
$$

By a similar proof of Theorem 4, we can obtain the following theorem.

Theorem 5. Let $f$ and $g$ be as defined in (1.5) and (2.5), respectively. Then, for $\lambda \geq 0,0 \leq \alpha<1,0<\beta \leq 1$ and $\frac{1}{2} \leq \gamma \leq 1$,

$$
f, g \in \Sigma_{p}(\lambda, \alpha, \beta, \gamma) \Longrightarrow \frac{1}{2} h \in \Sigma_{p}^{*}\left(\frac{(\lambda+1)^{2}(\lambda+2)^{2}(1+2 \beta \gamma-\beta)^{2}-16(\beta \gamma(1-\alpha))^{2}}{(\lambda+1)^{2}(\lambda+2)^{2}(1+2 \beta \gamma-\beta)^{2}+16(\beta \gamma(1-\alpha))^{2}}\right)
$$

where $h$ is given by (2.14). The result is sharp for the function given by (2.18). 


\section{Integral Transforms}

Theorem 6. Let $f$ be of the form (1.5) and $\lambda \geq 0$. If $f \in \Sigma_{p}(\lambda, \alpha, \beta, \gamma)$, then the integral transforms

$$
F_{c}(z)=c \int_{0}^{1} u^{c} f(u z) d u(0<c<\infty)
$$

are in the class $\Sigma_{p}\left(\lambda, \frac{2+c \alpha}{2+c}, \beta, \gamma\right)$. the result is sharp.

Proof. From the definition of $F_{c}$, we have

$$
F_{c}=c \int_{0}^{1} u^{c} f(u z) d u=\frac{1}{z}+\sum_{k=1}^{\infty} \frac{c a_{k}}{k+c+1} z^{k} .
$$

In view of Lemma 1 , it is sufficient to show that

$$
\sum_{k=1}^{\infty} B_{k}(\lambda) \frac{k(1+2 \beta \gamma-\beta)}{2 \beta \gamma\left(1-\frac{2+c \alpha}{2+c}\right)} \frac{c a_{k}}{k+c+1} \leq 1 .
$$

Since $f \in \Sigma_{p}(\lambda, \alpha, \beta, \gamma)$, (4.1) will be satisfied if,

$$
\frac{c}{\left(1-\frac{2+c \alpha}{2+c}\right)(k+c+1)} \leq \frac{1}{1-\alpha} .
$$

or equivalently, when

$$
I(k, \lambda, \alpha, c)=\frac{(1-\alpha) c}{\left(1-\frac{2+c \alpha}{2+c}\right)(k+c+1)} \leq 1 .
$$

Since $I(k, \lambda, \alpha, c)$ is a decreasing function of $k(k \geq 1)$, our proof is completed. The result is sharp for the function

$$
f(z)=\frac{1}{z}+\frac{2 \beta \gamma(1-\alpha)}{(1+2 \beta \gamma-\beta)(\lambda+1)(\lambda+2)} z .
$$

\section{Extreme Points and Its Application}

In view of Lemma 1 , we observe that $\Sigma_{p}(\lambda, \alpha, \beta, \gamma)$ is a closed convex family. Using Theorem $\mathbb{B}$ (or Lemma 1 ), we may obtain the extreme points of $\Sigma_{p}(\lambda, \alpha, \beta, \gamma)$.

Theorem 7. The extreme points of $\Sigma_{p}(\lambda, \alpha, \beta, \gamma)$, where $\lambda>-1,0 \leq \alpha<1$, $0<\beta \leq 1$ and $\frac{1}{2} \leq \gamma \leq 1$, are the functions given by

$$
F_{0}(z)=\frac{1}{z}, F_{k}(z)=\frac{1}{z}+\frac{2 \beta \gamma(1-\alpha)}{k B_{k}(\lambda)(1+2 \beta \gamma-\beta)} z^{k}(k=1,2, \ldots),
$$


where $B_{k}(\lambda)$ is defined in (2.2).

Proof. Since the operator $D^{\lambda}: \Sigma_{p}(\lambda, \alpha, \beta, \gamma) \rightarrow \Sigma_{p}(0, \alpha, \beta, \gamma)$ is an isomorphism from $\Sigma_{p}(\lambda, \alpha, \beta, \gamma)$ onto $\Sigma_{p}(0, \alpha, \beta, \gamma)$, it preserves extreme points. In view of $(2.3)$ and Theorem $\mathrm{B}$, it follows that the extreme points of $\Sigma_{p}(\lambda, \alpha, \beta, \gamma)$ are given by (5.1).

Corollary 5. Let $f$ be of the form (1.5), and $\lambda \geq 0$. If $f \in \Sigma_{p}(\lambda, \alpha, \beta, \gamma)$ then $\frac{1}{r}-\frac{4 \beta \gamma(1-\alpha)}{(1+2 \beta \gamma-\beta)(\lambda+1)(\lambda+2)} r \leq|f(z)| \leq \frac{1}{r}+\frac{4 \beta \gamma(1-\alpha)}{(1+2 \beta \gamma-\beta)(\lambda+1)(\lambda+2)} r(|z|=r)$, with equality for the function $F_{1}(z)$ defined in (5.1).

Proof. As a consequence of Theorem 7 , we have

$$
\frac{1}{r}-\max _{k} M(\lambda, \alpha, \beta, \gamma, k) r^{k} \leq|f(z)| \leq \frac{1}{r}+\max _{k} M(\lambda, \alpha, \beta, \gamma, k) r^{k},
$$

where

$$
M(\lambda, \alpha, \beta, \gamma, k)=\frac{2 \beta \gamma(1-\alpha)}{k B_{k}(\lambda)(1+2 \beta \gamma-\beta)} .
$$

It suffices to verify that $M(\lambda, \alpha, \beta, k)$ is a decreasing function of $k(k \geq 1)$, that is, that

$$
\frac{M(\lambda, \alpha, \beta, \gamma, k+1)}{M(\lambda, \alpha, \beta, \gamma, k)}=\frac{k(k+2)}{(k+1)(\lambda+k+2)} \leq 1
$$

which proves the result.

Remark. For $\lambda=0, \beta=1, \gamma=1$ and $\lambda=0$, Theorem 7 and Corollary 5 above give the corresponding results for $\Sigma_{p}(0, \alpha, 1,1)$ and $\Sigma_{p}(0, \alpha, \beta, \gamma)$, respectively.

\section{Acknowledgement}

This paper was supported by NON DIRECTED RESEARCH FUND, Korea Research Foundation, 1996.

\section{References}

[1] M. K. Aouf, "On a certain class of meromorphic univalent function with positive coefficients," Rend. Mat.(7) 11(1991), 209-219.

[2] S. K. Bajpai, "A note on a class of meromorphic univalent functions," Rev. Roumaine Math. Pures Appl. 22(1977), 295-297.

[3] N. E. Cho, S. H. Lee and S. Owa, "A class of meromorphic univalent functions with positive coefficients," Kobe J. Math. 4(1987), 43-50.

[4] J. Clunie, "On meromorphic schlicht functions," J. London Math. Soc. 34(1959), 215-216.

[5] J. E. Miller, "Convex meromorphic mappings and related functions," Proc. Amer. Math. Soc. 25(1970), 220-222. 
[6] M. L. Morgra, T. R. Reddy and O. P. Juneja, "Meromorphic univalent functions with positive coefficients," Bull. Austral. Math. Soc. 32(1985), 161-176.

[7] Ch. Pommerenke "On meromorphic starlike functions," Pacific J. Math. 13(1963), 212-235.

[8] M. S. Robertson, "Convolutions of Schlicht functions," Proc. Amer. Math. Soc. 13(1962), 585-589.

[9] B. A. Uralegaddi and M. D. Ganigi, "A new criterion for meromorphic convex functions," Tamkang. J. Math. 19(1988), 43-48.

Department of Mathematics, Kyungsung University, Pusan, 608-736, Korea

(E-mail: oskwon@cpd.kyungsung.ac.kr)

Department of Applied Mathematics, Pukyong National University, Pusan 608-737, Korea

(E-mail: necho@delphin.pknu.ac.kr) 